\title{
Measuring Cognitive Load using Eye Tracking Technology in Visual Computing
}

\author{
Johannes Zagermann, Ulrike Pfeil, and Harald Reiterer \\ HCI Group, University of Konstanz \\ \{johannes.zagermann,ulrike.pfeil,harald.reiterer\}@uni-konstanz.de
}

\begin{abstract}
In this position paper we encourage the use of eye tracking measurements to investigate users' cognitive load while interacting with a system. We start with an overview of how eye movements can be interpreted to provide insight about cognitive processes and present a descriptive model representing the relations of eye movements and cognitive load. Then, we discuss how specific characteristics of human-computer interaction (HCI) interfere with the model and impede the application of eye tracking data to measure cognitive load in visual computing. As a result, we present a refined model, embedding the characteristics of HCI into the relation of eye tracking data and cognitive load. Based on this, we argue that eye tracking should be considered as a valuable instrument to analyze cognitive processes in visual computing and suggest future research directions to tackle outstanding issues.
\end{abstract}

\section{ACM Classification Keywords}

H.5.2. User Interfaces: Input devices and strategies (e.g., mouse, touchscreen). Evaluation/methodology

\section{Author Keywords}

cognitive load; eye tracking; novel evaluation methods

\section{INTRODUCTION}

In addition to classical measurements of usability (effectiveness, efficiency, satisfaction) [10], researchers and practitioners in visual computing are constantly investigating additional metrics to assess the quality of system support for human task performance. One such metric that is currently of interest is the cognitive load that the system and task place on the user. Cognitive load describes the amount of mental effort that is used from the working memory while performing a cognitive task [5] and interacting with a system [22]. Designing successful systems means implementing visualizations and interaction techniques in a way that they require little capacity to use and allow the user to concentrate on the task at hand. This includes to make good use of people's perceptual and cognitive abilities.
Focusing on cognitive processes, Sweller [23] distinguished three types of load. The first kind of cognitive load is known as intrinsic load, which is directly connected to the task at hand (e.g. formulating a text). It cannot be influenced or manipulated by the designer of a system. Extraneous load refers to the load that is imposed by the format and the way in which information is presented (e.g. the structure of an interface). Germane load is caused by the effort that has to be made in order to understand and process the materials. The goal of successful visualizations is thus to keep the extraneous load to a minimum by providing smooth system interaction. A low extraneous load leads to more cognitive capacities for intrinsic and germane load that both help to solve the task at hand.

One widely used and validated measurement for the analysis of task load is the NASA task-load index (TLX) [9] questionnaire, which divides task load that a system or a task places on users into six sub-aspects: mental demand, physical demand, temporal demand, own perception of performance, effort and frustration. This questionnaire is currently the standard measure to investigate the extent to which a system or a task strains users mentally, physically and emotionally. It is widely applied in the HCI and information visualization communities $[8,1]$. As the nature of questionnaires applies, it relies on subjective ratings of participants after an experiment. Thus, in situ information cannot be provided, which implies that the NASA-TLX cannot be used to provide real time information e.g. for adapting an interface to the current load of a user. In addition, the NASA-TLX collects users' subjective opinions, and it might not be applicable to assess unconscious and even automatic processes.

Traditionally, brain activity has been measured through techniques such as electroencephalography (EEG) and magnetoencephalography (MEG) whose objective is to capture changes in magnetic fields at the scalp caused by changing electrical currents in brain neurons [7]. Cognitive load is one of the indications of brain activity. The main strength of these techniques is their millisecond-level time precision. However, this measurement is quite intrusive to the user, requires a timeconsuming setup and its analysis is often complex. This also inhibits real-time information and can therefore not be used in situ or even in public. There are many other non-neural techniques that are related to brain activity and cognitive load. Cortical activity causes a small nervous response that is translated into variations in heart rate, blood pressure, electrodermal activity, electrical activity in facial muscles, eye movements and small dilations of the pupil. 
Recent studies have employed eye movement measures to investigate cognitive processes [17]. To this end, several studies have investigated the relation of voluntary eye-movements like fixations and saccades on cognitive processes [21] as well as involuntary eye-movements like blink rate and pupil dilation [17]. These eye movements are also called behavioural (voluntary) and physiological (involuntary) [5]. Through the tracking of the eyes, one can study a user's physical reaction to a task and a system and adapt the interface accordingly. For example, in controlled environments, high-precision eye trackers and pupillometers can be used to detect small pupil dilations that are indicators of cognitive load [20]. However, information visualization systems cover a wide span of content, visual representations and interaction modalities which in itself influence users' eye movements and make it difficult to directly use eye movements as a measure of cognitive load in situ. Thus, it is important to identify the interferences between eye movements and cognitive load.

We believe that aspects of human-computer interaction can help us understand how system interaction influences eye movements. Especially in the visualization of large datasets interactivity and thus HCI can be crucial factors: Interactivity allows to manage limitations either on the human or on the computational side by e.g. providing the possibility to investigate multiple levels of detail, to switch between different views, or to compare various representations of data simultaneously [16]. Therefore, the field of HCI provides theories and tools that help to design user interfaces that focus on human factors as well as on the integration of computational power [13]. A model describing the relation of eye movement, aspects of human-computer interaction and cognitive load can help us understand how eye movements can be taken as a measure of cognitive load in visual computing.

Current laptops have cameras to track faces, thus a technical implementation of eye tracking technology into interactive systems is becoming standard. If there is a link between cognition and eye movements, eye tracking data can be used to detect users' cognitive states in situ. This information can then be used by the system to adapt characteristics of visualization and interaction to user's current cognitive capacities (like suggested by Portas et al. [19] and Manuel et al. [15] in the learning domain). Thus, eye tracking could be used to measure users' cognitive processes during system usage to allow the system to adapt to users current cognitive load.

\section{Goal of the paper}

Traditionally, performance has been described as the completion time of a task and the error rates. Including a third measurement, cognitive load, can complement our definition of performance. It will offer the possibility to distinguish between users who perform a task with equal completion time and error rates but with different levels of mental effort, helping to develop interfaces that require less cognitive capacities. In addition, real-time information about a user's cognitive load could be used to adapt the content and presentation of a system.
The aim of this position paper is to discuss the applicability of eye tracking data to study cognitive load. This goal can be divided into the following sub-questions:

- What are the eye tracking measures that are applicable to analyze cognitive load in visual computing? (RQ1)

- Which aspects of Human-Computer Interaction influence cognitive load? (RQ2)

- How can we analyze cognitive load in visual computing using eye tracking? (RQ3)

We aim to construct a model that describes the link between eye tracking metrics and cognitive load. In addition, we discuss how characteristics of HCI interfere with this model and revise it respectively. We see this revised model as both, a starting point for future research on this topic as well as common ground, which can trigger inspiration and discussions. Findings can then be used to validate and further refine this model iteratively. Finally, we suggest future work of what we think could be either potential studies or ideas dealing with real-world influences analyzing cognitive load with eye tracking.

\section{EYE MOVEMENTS AND COGNITIVE LOAD}

This section summarizes eye tracking measures in general and their relevance in assessing cognitive load. We discuss related studies that employed eye tracking metrics in order to investigate users' cognitive and emotional processes. In the second part, we present our model, describing the relations of eye tracking metrics and cognitive load.

\section{Eye tracking measures}

As mentioned above, eye movements can be studied to detect users' cognitive states. This can be used to provide a system with information about the user's cognitive activities. In the following section, we present related work to illustrate how eye tracking measures are used to investigate and interpret cognitive processes and cognitive load. We distinguish between voluntary eye-movements like fixations and saccades and involuntary eye-movements like pupil dilation and blinking. In the following, these measurements are discussed and their relation to cognitive processes and cognitive load is elaborated on.

\section{Fixations}

The most common type of eye tracking event refers to a focused state when the eye remains still over a period of time. This event is called fixation and lasts from 200-300 milliseconds to up to several seconds. It is a voluntary movement. The number of fixations indicates the number of times that a user looked to a certain area of interest (AOI). Thus, the state of attention is indicated by the position of the gaze within the display or a specific AOI. Rudmann et al. [21] found that the gaze direction indicates the interface element that is relevant for the current cognitive activity. It can be interpreted as a repeated interest in an area. One can also make use of the fixation duration, the period of time that a user looked to a certain AOI. The fixation duration has been related to the level of cognitive processing with a high fixation duration indicating 
an increased strain on the working memory. This was accompanied by a decrease in fixation rate. Chen et al. conclude that fixation duration and fixation rate are indicators of an increment in the attention that is needed as the complexity of the task increases [6].

Based on these findings, we propose the influence of cognitive load on fixation duration (the higher the load, the longer the fixations) and fixation rate (the higher the load, the lower the fixation rate).

\section{Saccades}

Another eye tracking event refers to a shift between two locations. When the eye performs a voluntary movement from one fixation to another, this event is called saccade. This movement is the fastest movement the body can produce and typically takes from 30-80 milliseconds to complete. The most common visualization for saccades are scanpaths. One can measure the velocity and length of saccades and observe the patterns of a scanpath. Chen et al. [6] included the measurements of saccade velocity and saccade length in order to investigate human mental effort. Amongst other measures, their results show that saccade velocity and length are highly discriminatory parameters, both related to achieving a high performance. Similarly, Manuel et al. [15] have found that a decrease in saccade velocity indicates tiredness and an increase of saccade velocity indicates a higher task difficulty.

Based on these findings, we propose an influence of cognitive load on saccade length (the higher the load, the longer the saccades) and saccade velocity (the higher the load the higher the saccade velocity): However, we have to take into account that these two measurements are highly correlated.

\section{Pupil dilation}

Another relevant eye measurement is the pupillary response. It is an involuntary reflex and the pupil can range in diameter from $1 / 16$ inch $(1.5 \mathrm{~mm})$ to more than $1 / 3$ inch $(8 \mathrm{~mm})$. Psychologists have argued for more than twenty years that changes in pupil dilation accompany effortful cognitive processing. Previous research has shown that users' pupils dilate when the difficulty of the task and their cognitive effort to solve it increase $[6,19,20,18,14]$. Many studies have validated this argument across a variety of tasks, including reading, problem solving, and visual tasks [21]. Porta et al. [19] also observed a decrease in pupil diameter size towards the end of the task in their experiment, interpreting it as a potential sign for tiredness. In addition to cognitive processes, variations in the brightness of the environment produces changes on the pupil size. When the environment becomes darker, the pupil dilates in order to acquire more light and it contracts when the environment becomes brighter. Controlling environmental brightness and display luminance is crucial when pupil dilation is investigated in experiments. Pomplun and Sunkara [18] have developed and implemented an eye tracker calibration interface that is able to almost completely eliminate the geometry-based distortion of pupil measurements. They perform a pupil calibration setup to determine the relative size of the pupil as a function of the participant's gaze position. In order to compute the pupil dilation induced by cognitive load, they subtract the calibration value for the current display brightness from the current measured pupil size resulting in a method for accurate cognitive workload measurement even in situations where the display brightness cannot be kept constant.

Therefore, we propose an influence of cognitive load on users' pupil sizes, in the sense that the pupil dilates with increasing cognitive load.

\section{Blink rate}

Rate and latency of blinking activities can be another eye tracking metric providing information about a user's cognitive load. Blinking can be a voluntary eye movement although, most of the time, it is an involuntary movement. The rate and latency of blinks can help to provide a deeper understanding about the state of attention of the participant. For example, high blink latency and a low blink rate has been found to indicate high mental effort [6]. Similarly, Manuel et al. [15] found that an increase in the blinking rate, a decrease in the blinking velocity and a decreasing degree of the eyelids' openness are signs of increasing tiredness.

Based on these findings, we propose an influence of cognitive load on blink rate (the higher the load, the lower the blink rate) and blink latency (the higher the load, the higher the blink latency). Furthermore, there might be a relation between cognitive load and blink velocity.

\section{Model of Eye Movements and Cognitive Load}

There have been several studies in the field of cognition states and eye tracking for visual and auditive tasks, e-learning, and visualization of interfaces that investigate cognitive processes and cognitive load. This previous research shows that voluntary as well as involuntary eye movements can be used as ways to measure cognitive load. Figure 1 shows the influence of cognitive load on aspects of the four discussed eye movements: fixations, saccades, pupil dilation, and blinks. For the sake of simplicity, we deliberately focused on these main metrics. Future research has to prove if minor eye movements like smooth pursuit or microsaccades could be indicators of cognitive load, too.

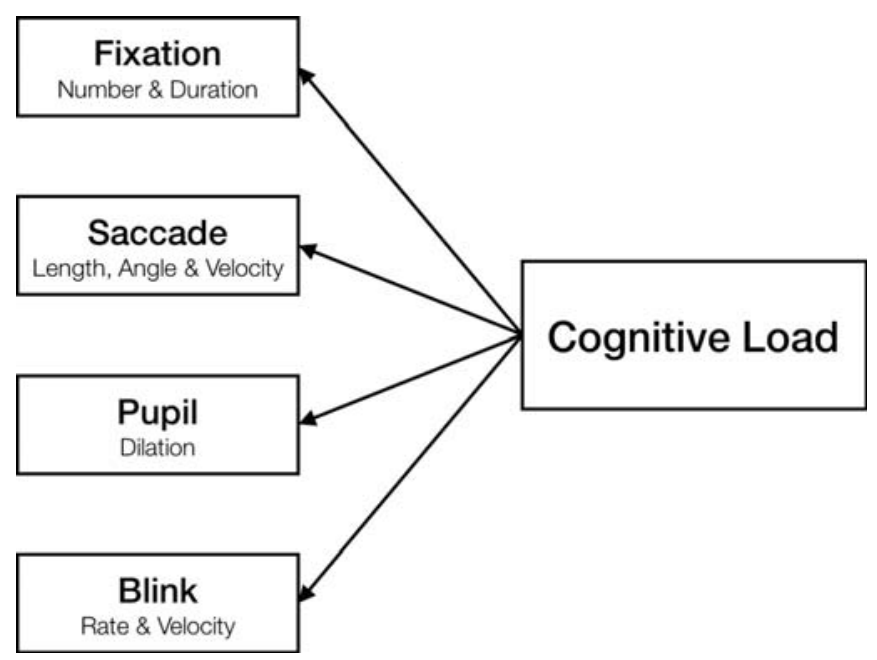

Figure 1. Model of eye-based indicators of cognitive load. 
As previously discussed, our model (see Figure 1) is based on several mostly artificial lab experiments with e.g. controlled luminance of the environment and the interface, a precise sequence of tasks, and high precision devices. This results in a focus on intrinsic (represented by a task) and germane (processing of content) load. We believe that in real-world visualization settings, it is especially important to focus on extraneous factors as a minimization of these can provide free cognitive capacities to be used for the task at hand. At the moment, extraneous factors, that are essential aspects of HCI, are mostly excluded: Visualization and Interaction. To further elaborate on this, we discuss several aspects of human-computer interaction based on the conceptual framework Blended Interaction [11] and their influence on eye movements in the following section.

\section{BLENDED INTERACTION \& DOMAINS OF DESIGN}

Jetter et al. [11] introduced Blended Interaction - a conceptual framework that describes the nature of human-computer interaction. The concept of Blended Interaction combines the virtues of familiar physical and social environments with the benefits of the digital realm in a way that desired properties of both worlds are preserved while providing a 'natural' human-computer interaction. To illustrate their concept of Blended Interaction, Jetter et al. [11] apply it to four domains of design: Individual Interaction, Social Interaction and Communication, Workflow, and Environment. These four domains can basically serve as lenses or points of view to identify HCI aspects of information visualizations.

- Individual Interaction describes the way each individual interacts with an interactive system, the usage of different input modalities like pen, touch, or traditional input via mouse but also the interface itself.

- Social Interaction and Communication describes the social aspects, standards or norms that influence the way we collaborate via the system.

- Workflow describes the overall workflow in which multiple tasks are embedded.

- Environment describes the physical environment in which interaction takes place.

The framework and especially the named four domains encompass not only technical aspects but also physical surroundings and social context of an interface. As these additional factors also influence eye movements, we believe that this framework - although it was originally developed for a different purpose is particularly applicable for our work.

\section{Eye tracking and Blended Interaction}

In this section we describe possible correlations between the four domains of Blended Interaction and major eye movements.

\section{Individual Interaction}

Visual interfaces are designed to draw users' attention to specific areas of interest: menus, content, and tools. The task at hand requires users to focus on specific content while knowing where to find proper tools. Thus, the location of the fixation is influenced by the system design in the sense that the content influences the users' fixations. Visual representations of content might influence fixation location, duration and rate without a cognitive engagement.

The structure of the interface as well as the activity that the user performs with the system influence saccadic eye movements: for example in visual analytic tasks where users have to switch between different tools, layers, and views, users not only have to select and use the named interface elements, but also have to visually search for specific items. Depending on the structure of the interface and the expertise of the user, this can result in differences in length, velocity and angle of saccades. Thus, the activity that the user engages in influences saccadic eye movements. For example, searching for information in images results in different saccades than reading a text.

Interaction with a system through different in- and output technologies and modalities also influence eye movements. Input devices might require the user to not only fixate on visual information on a display, but also to visually focus on the input device itself. Knowledge worker often switch between different media: Printed information has to be read, highlighted with physical tools such as pens and later summarized on digital input and output devices. Visual analysts often switch between desktop displays, high-resolution wall displays and portable devices while taking notes on sheets of paper [13]. This mixedfocus working style with multiple analog and digital media artifacts and devices influences saccadic eye movements and fixations. In addition, it also influences pupil dilation and blinking behavior as the luminance of the element in focus changes frequently.

In summary, we conclude that regarding the individual interaction, it is especially the content, the activity, as well as the input- and output modality that influence eye movements (see column b) in Figure 2). Thus, it is important to focus on these kinds of interferences when investigating the relation between eye movements and cognitive load through the lens of individual interaction.

\section{Social Interaction and Communication}

Many interactive systems are inherently social, as users often work with a system in groups. Even in cases when a user uses the system individually, (s)he is situated in a social context, as (s)he is embedded in a social environment which might require him/her to engage in communication and coordination activities beyond system usage. This means, that users might change their visual focus often, in order to cope with social aspects while interacting with an application. Depending on the activity, the number of collaborators, and interactive devices various physical as well as social situations can occur. For example, multiple persons can stand around a tabletop, gather around a small display, or sit in rows while another person holds a presentation.

Different physical positions of persons as well as social roles can influence saccadic eye movements to allow for face-to-face communication. Switching focus between different collaborators and interactive devices not only results in differences in fixations and saccades but also in differences in blinking rates 


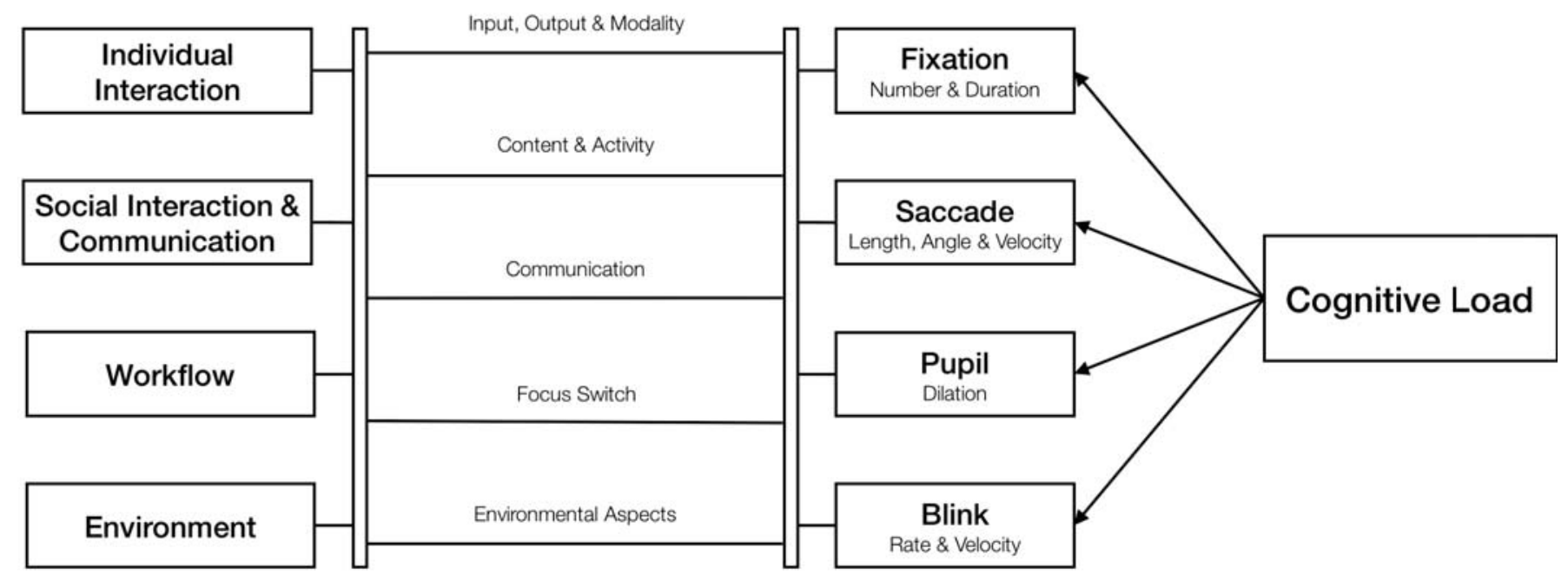

a)

b)

c)

d)

Figure 2. Model of eye-based indicators of cognitive load with possible influences of different aspects of HCI.

and its velocity. This mixed-focus nature of collaboration can be exhausting for the eye itself, resulting in a higher blink rate. Communication with collaborators often appears as face-toface communication, which means focusing on another person. In combination with working on digital tabletops, or mobile devices, the pupil size changes due to different luminances and distances.

Investigating the cognitive load with eye tracking requires to take into account the social setting as well. Analyzing systems through the lens of Social Interaction and Communication requires to take into account the interference of eye movements through communication (see column b) in Figure 2).

\section{Workflow}

The structure and sequence of individual and social interaction activities can be guided by an overall workflow that defines several phases of a task and the intended outcome. Often, these workflows can be described as mixed-focus: Knowledge workers find important information in physical books on their table, that have to be transferred to office applications, using the right tools and input devices. Same for visual analysts: the comparison of documents and visualizations [3], the summarization of interesting data [4], and possible discussions with colleagues [12] can be seen as subtasks of an overall workflow.

Multiple devices and pieces of information in a variety of media are represented as various areas of interest and should thus be considered when analyzing cognitive load in visual computing. The sequence of various tasks and activities in mixed-focus environments can require the user to focus on different areas of interest (either digital or analog) and therefore influence saccadic eye movements. The same is true for different subtasks: Switching between printed and digital media or multiple devices with different screens can be exhausting and thus influence blinking behavior. Also, different types of media or even different types of output devices can have various luminances, which influences pupil dilation.

When analyzing cognitive load based on eye tracking data in settings that require users to engage in multiple activities with multiple devices, researchers should take into account that users have to switch their visual focus frequently. Thus, it is important to incorporate focus switch (see column b) in Figure 2) as a main interference when analyzing cognitive load with eye tracking through the lens of Workflow.

\section{Environment}

Interactions take place in a physical environment: e.g. an office, a control room, or in a conference room. Different types and shapes of interactive devices and a great variety of physical surroundings (either moving or stationary) compete for users' attention and focus while performing a task. Thus, an interface cannot be regarded on its own, but the resulting fixations have to be evaluated with respect to the physical surroundings. Not only multiple different devices and the resulting switch of focus but also environmental characteristics like e.g. air temperature, humidity, or luminance can exhaust the eye and result in blinking. The physical environment and the overall luminance have the highest impact on pupil size. Eyes regulate light input depending on the luminance of an environment with changing the size of pupils.

When analyzing cognitive load based on eye movements through the lens of Environment, it is thus important to incorporate environmental aspects (see column b) in Figure 2) into the analysis as it poses several interferences to the eye tracking measurements.

\section{A Revised Model of Eye Movements and Cognitive Load}

Based on the discussion of the four domains of HCI in relation to eye movements, we revised our model. Figure 2 
shows how the four domains of design directly influence eye movements. The individual interaction, which refers to different input \& output devices as well as modalities, has an impact on number $\&$ duration of fixations - the content and the current activities influence different aspects of saccadic eye movements. Social roles and the physical position of involved persons and thus communication can lead to pupil dilation as the focus changes. Environmental aspects can also influence pupil size and blinking rates e.g. due to factors like luminance or humidity.

We believe that this model can serve as a starting point for future research when measuring cognitive load. It shows the influences of system usage on eye movements that need to be taken into account when using the same eye movements to interpret users' cognitive load. As we are in the starting phase of a research project on quantitative methods for visual computing ${ }^{1}$, we want to further evaluate, iterate, and extend our proposed model.

As a first step, we present possible studies and ideas on how to deal with real-world influences on eye tracking measurements of cognitive load in the next chapter.

\section{IMPLICATIONS FOR FUTURE RESEARCH}

The following four implications for future research serve as both: starting points for further evaluations as well as examples of how the influences of different confounding factors can be addressed when assessing users' cognitive load using eye tracking technology.

\section{Pupil Dilation and Environment}

Pfleging et al. [17] proposed a model on how to measure workload based on pupil dilation independently of the environmental luminance. In their experiment, they studied the influence of six different lighting conditions and tasks with varying mental demand. In their model, they describe the total pupil dilation as the sum of luminance-based and workload-based influence. Thus, knowing the luminance of the environment can help to calculate load originated by the task at hand (see Figure 3). However, as individual differences in pupil size are quite large, one cannot easily substract the luminace-based value from an individual's pupil diameter without knowing the individual pupil diameter in a non-loaded situation. This could be solved by calculating an individual's change of pupil diameter as the measured luminance (interface, output device, and environment) varies over time while interacting with a system. These values can then be used to establish an individual's average pupil size and how it reacts to changes in light conditions (e.g. compare the machine learning based classification of cognitive load as described by [5]). Based on this, conclusion about the user's cognitive load could be drawn, not based on a static value but based on the pupil size's dynamic changes over time.

Future research into this should focus on real-time measurements of pupil dilation and light condition and on how these values can be taken as a baseline to conclude about the user's cognitive demand ad hoc.

\footnotetext{
${ }^{1} \mathrm{SFB} /$ Transregio 161 - http://www.trr161.de
}

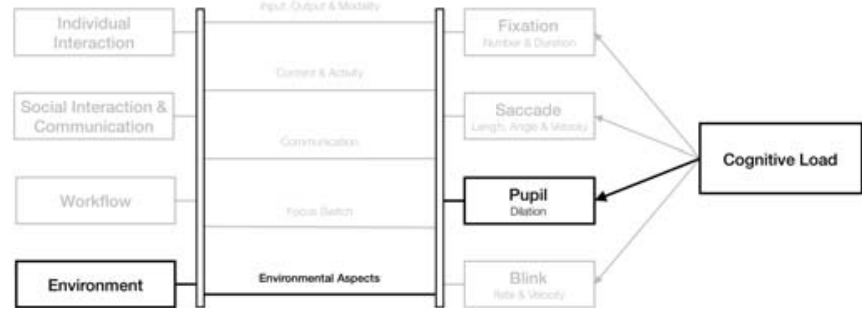

Figure 3. Focus on the relation of Environment and Pupil Dilation

\section{Saccades and Individual Interaction}

Saccadic eye movements are highly distinctive and taskdependent [24]. Based on the task at hand and the content of the interface, a model could predict appropriate saccadic eye movements (see Figure 4). For example, when reading a document, it is already possible to tell from the patterns of a scanpath how well the reader has understood the text [2]. Aligning the actual saccadic eye movements with the ideal saccades for a specific text helps to identify the extent to which the user has understood the text. In addition, the number of regressions (times that a user's saccades go backwards whilst reading) as well as the reading speed (based on the time between the start and end fixation of reading) indicate users' comprehension of a text. Based on this knowledge, conclusions can be drawn about users' current state of cognitive load. This information could be used in real-time e.g. to lower the difficulty of the text (if the cognitive load is high) or provide additional information such as further descriptions, or annotations (to challenge the user if the cognitive load was considered to be low).

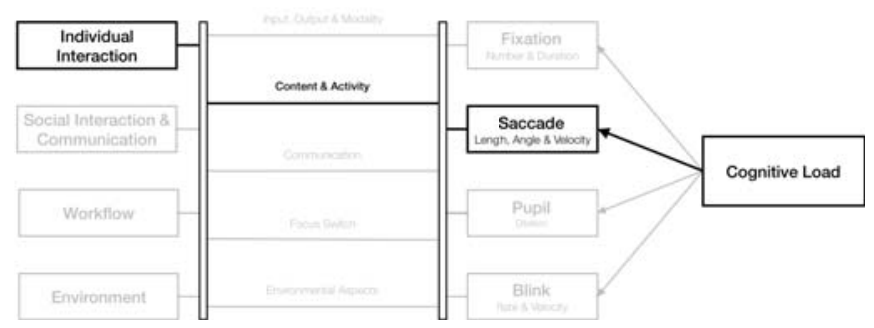

Figure 4. Focus on the relation of Individual Interaction and Saccades

Future research should thus aim at investigating "optimal" saccadic patterns for specific atomic and compound tasks (like has been done for reading) that are considered to be essential in visual computing settings (for example analyze, produce, search, query [16]). Furthermore, future research should address how these optimal paths could be taken as a baseline for comparisons with actual scanpaths to indicate a user's cognitive load.

\section{Fixations and Individual Interaction}

Up to now, researchers have mainly investigated AOIs when using eye tracking to evaluate interactive systems. Further, Rudmann et al. [21] have used the investigation of AOIs in relation to cognitive states. Their results show that users actually visually focus on relevant parts of the system when solving a task. Depending on the content and the task at hand, 
focusing on specific aspects might support the user in its task and therefore lower his cognitive effort. One example of this is the attention that the user has to place on the input device instead of the display (see Figure 5). Ideally, interaction with the input device should be easy and smooth and should not require any visual focus from the user's side. Thus, measured eye-fixations on the input device could indicate that handling the device places an unnecessary cognitive demand on the user. This can also be transferred onto the visual display as well. Not being stressed might result in the user fixating AOIs that help him performing a task. Cognitive load might trigger him in visually attending to AOIs that are not of importance in his situation. Also, the distribution of fixations on the display could indicate cognitive load, as frequent fixation-jumps between different AOIs might indicate a higher cognitive load than visually attending to one AOI (many fixations within one AOI) before moving to the next one. On the other side, focusing too long on one AOI might also indicate that the user has difficulties understanding the content and is cognitively loaded.

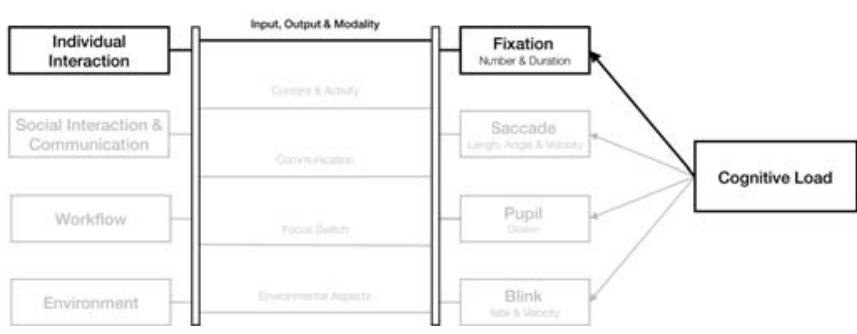

Figure 5. Focus on the relation of Individual Interaction and Fixations

Future research should further address the relation of fixation duration time on an AOI and cognitive load in different task settings to better understand how dwell time and fixation rate as well as spatial distribution of fixations can be taken as a measurement of cognitive load in visual computing.

\section{Blinks and Workflow \& Environment}

Both, the workflow for the tasks at hand and the environment in which users perform them, can influence the blinking rate. Different lighting conditions and brightnesses of output devices can exhaust the eye, which leads to a higher blinking rate (see Figure 6). This increased blinking rate could be falsely interpreted as an indicator for cognitive load as up to now it is not possible to detect whether a blink is originated by the cognitive load a task places on the users or whether it is caused by workflow sequences with mixed-focus tasks. Using mobile eye tracking glasses, a system could match the current gaze position to specific context information like e.g. the brightness of the current display or the type of media. Blinks that are detected as not related to cognitive load could then be dismissed. As the blinking rate is not only highly depending on the task and environment but also on the individual eye characteristics, a running average per user could detect blinking patterns regarding the workflow and environment. Based on this, a system could detect blinks connected to an increased cognitive load and adapt the content of the current task accordingly. Additional recommendations could be served to help users to be aware of their actual load.

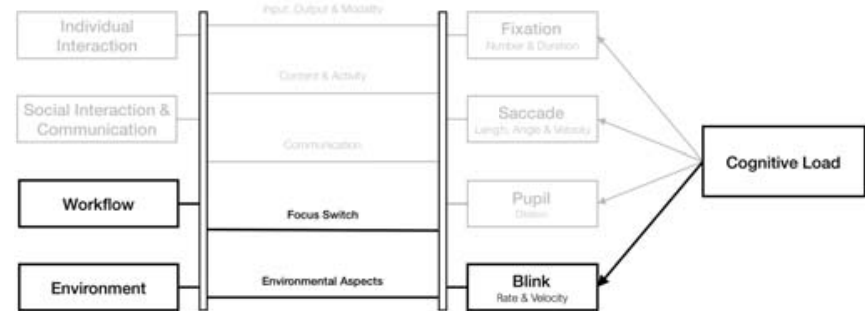

Figure 6. Focus on the relations of Workflow, Environment, and Blinks

Future research should thus further investigate factors that can exhaust eyes like e.g. brightness of displays or similar to further create models that describe their influence.

We think that these four described implications for future research could serve as triggers as well as inspiration of how different aspects of human-computer interaction can influence the way cognitive load can be measured using eye tracking technology. We think that a combination of multiple measurements of eye movements with respect to the task at hand and the overall context is a promising way to cope with current issues.

\section{CONCLUSION}

Measuring cognitive load using eye tracking technology can be seen as an effective and convenient way to evaluate systems beyond classical measurements such as errors or task completion time. The provided data is objective and can be accessed in real time, which allows to adapt interfaces to the current cognitive load of a user. Our descriptive model represents correlations between major eye movements and cognitive load (RQ1). We discussed possible influences and effects based on the four domains of design of the conceptual framework Blended Interaction, which lead us to a revised model of the relation of eye movements, cognitive load and human-computer interaction (RQ2). The implications for future research show possible starting points for further investigations for outstanding issues $(\mathbf{R Q 3})$.

Overall we conclude that eye tracking can be a valuable instrument to measure and analyze cognitive load in the context of HCI and visual computing. In future we want to further evaluate, refine, and extend our proposed model - our vision is a tool that supports researchers equally in designing experiments and explaining phenomena in the intersection of eye tracking and cognitive load.

\section{ACKNOWLEDGMENTS}

We thank the German Research Foundation (DFG) for financial support within project C01 of SFB/Transregio 161. We also thank Lewis Chuang, Jakob Karolus, Valentin Schwind, and Elena Barreras-Aragon for their valuable comments and ideas.

\section{REFERENCES}

1. Erik W. Anderson. 2012. Evaluating Visualization Using Cognitive Measures. In Proceedings of the 2012 BELIV Workshop: Beyond Time and Errors - Novel Evaluation 
Methods for Visualization (BELIV'12). ACM, New York, NY, USA, 1-4.

2. David Beymer and Daniel M. Russell. 2005. WebGazeAnalyzer: A System for Capturing and Analyzing Web Reading Behavior Using Eye Gaze. In CHI '05 Extended Abstracts on Human Factors in Computing Systems (CHI EA '05). ACM, New York, NY, USA, 1913-1916.

3. Matthew Brehmer and Tamara Munzner. 2013. A Multi-Level Typology of Abstract Visualization Tasks. IEEE Trans. Visualization and Computer Graphics (TVCG) (Proc. InfoVis) 19, 12 (2013), 2376-2385.

4. Matthew Brehmer, Michael Sedlmair, Stephen Ingram, and Tamara Munzner. 2014. Visualizing Dimensionally-reduced Data: Interviews with Analysts and a Characterization of Task Sequences. In Proceedings of the Fifth Workshop on Beyond Time and Errors: Novel Evaluation Methods for Visualization (BELIV'14). ACM, New York, NY, USA, 1-8.

5. Fang Chen, Jianlong Zhou, Yang Wang, Kun Yu, Syed Z. Arshad, Ahmad Khawaji, and Dan Conway. 2016. Robust Multimodal Cognitive Load Measurement. Springer International Publishing.

6. Siyuan Chen, Julien Epps, Natalie Ruiz, and Fang Chen. 2011. Eye Activity As a Measure of Human Mental Effort in HCI. In Proceedings of the 16th International Conference on Intelligent User Interfaces (IUI '11). ACM, New York, NY, USA, 315-318.

7. Jérémy Frey, Maxime Daniel, Julien Castet, Martin Hachet, and Fabien Lotte. 2016. Framework for Electroencephalography-based Evaluation of User Experience. In Proceedings of the 2016 CHI Conference on Human Factors in Computing Systems (CHI'16). ACM, New York, NY, USA, 2283-2294.

8. Sandra G. Hart. 2006. NASA-task load index (NASA-TLX); 20 years later. In Proceedings of the human factors and ergonomics society annual meeting, Vol. 50. Sage Publications, 904-908.

9. Sandra G. Hart and Lowell E. Stavenland. 1988. Development of NASA-TLX (Task Load Index): Results of empirical and theoretical research. In Human Mental Workload. Elsevier, Chapter 7, 139-183.

10. Kasper Hornbæk. 2006. Current Practice in Measuring Usability: Challenges to Usability Studies and Research. Int. J. Hum.-Comput. Stud. 64, 2 (Feb. 2006), 79-102.

11. Hans-Christian Jetter, Harald Reiterer, and Florian Geyer. 2014. Blended Interaction: Understanding Natural Human-computer Interaction in post-WIMP Interactive Spaces. Personal Ubiquitous Comput. 18, 5 (June 2014), 1139-1158.

12. Youn-ah Kang and John Stasko. 2012. Examining the Use of a Visual Analytics System for Sensemaking Tasks: Case Studies with Domain Experts. IEEE Transactions on Visualization and Computer Graphics 18, 12 (2012), 2869-2878.

13. Daniel Keim, Gennady Andrienko, Jean-Daniel Fekete, Carsten Görg, Jörn Kohlhammer, and Guy Melançon. 2008. Information Visualization. Springer-Verlag, Berlin, Heidelberg, Chapter Visual Analytics: Definition, Process, and Challenges, 154-175.

14. Jeff Klingner, Rakshit Kumar, and Pat Hanrahan. 2008. Measuring the Task-evoked Pupillary Response with a Remote Eye Tracker. In Proceedings of the 2008 symposium on Eye tracking research \& applications. ACM, 69-72.

15. Victor Manuel, Victor M. Garcia-Barrios, Christian Gütl, Alexandra Preis, Keith Andrews, Maja Pivec, Felix Mödritscher, and Christian Trummer. 2004. AdELE: A Framework for Adaptive E-Learning through Eye Tracking. In Proceedings of IKNOW 2004. 609-616.

16. Tamara Munzner. 2014. Visualization Analysis and Design. A K Peters.

17. Bastian Pfleging, Drea K. Fekety, Albrecht Schmidt, and Andrew L. Kun. 2016. A Model Relating Pupil Diameter to Mental Workload and Lighting Conditions. In Proceedings of the 2016 CHI Conference on Human Factors in Computing Systems (CHI '16). ACM, New York, NY, USA, 5776-5788.

18. Marc Pomplun and Sindhura Sunkara. 2003. Pupil Dilation as an Indicator of Cognitive Workload in Human-Computer Interaction. Proceedings of the International Conference on HCI (2003), 542-546.

19. Marco Porta, Stefania Ricotti, and Calet Jimenez Perez. 2012. Emotional E-Learning through Eye Tracking. In Global Engineering Education Conference (EDUCON), 2012 IEEE. IEEE, 1-6.

20. Sohail Rafiqi, Chatchai Wangwiwattana, Jasmine Kim, Ephrem Fernandez, Suku Nair, and Eric C. Larson. 2015. PupilWare: Towards Pervasive Cognitive Load Measurement Using Commodity Devices. In Proceedings of the 8th ACM International Conference on PErvasive Technologies Related to Assistive Environments (PETRA '15). ACM, New York, NY, USA, 1-8.

21. Darrell S. Rudmann, George W. McConkie, and Xianjun Sam Zheng. 2003. Eyetracking in Cognitive State Detection for HCI. In Proceedings of the 5th International Conference on Multimodal Interfaces (ICMI '03). ACM, New York, NY, USA, 159-163.

22. John Sweller. 1988. Cognitive Load During Problem Solving: Effects on Learning. Cognitive Science 12, 2 (1988), $257-285$.

23. John Sweller. 1999. Instructional Design in Technical Areas. ACER Press.

24. Alfred L. Yarbus. 1967. Eye Movements and Vision. Plenum. New York. 